\title{
Study of efficient homogenization algorithms for nonlinear problems
}

\author{
Approximation of a homogenized tangent stiffness to reduce computational cost
}

\author{
Jun-ichi Okada · Takumi Washio • Toshiaki Hisada
}

Received: 30 January 2009 / Accepted: 10 October 2009 / Published online: 31 October 2009

(C) The Author(s) 2009. This article is published with open access at Springerlink.com

\begin{abstract}
A framework for the homogenization of nonlinear problems is discussed with respect to block LU factorization of the micro-macro coupled equation, and based on the relation between the characteristic deformation and the Schur-Complement as the homogenized tangent stiffness. In addition, a couple of approximation methods are introduced to reduce the computational cost, i.e., a simple scheme to reuse the old characteristic deformation and a sophisticated method based on the mode-superposition method developed by our group. Note that these approximation methods satisfy the equilibrium conditions in both scales. Then, using a simplified FE model, the conventional algorithm, a relative algorithm originating from the block LU factorization, and the above-mentioned algorithms with the approximated Schur-Complement are compared and discussed. Finally, a large-scale heart simulation using parallel computation is presented, based on the proposed method.
\end{abstract}

Keywords Homogenization method · Nonlinear finite element analysis - Schur-Complement - Mode superposition · Parallel computation · Block LU factorization . Heart

\section{List of symbols}

$\mathbf{Y}, \mathbf{y}$ Position vector around the deformation in the microstructure

$\mathbf{X}, \mathbf{x}$ Position vector around the deformation in the macrostructure

J. Okada $(\varangle) \cdot$ T. Washio · T. Hisada

Graduate School of Frontier Sciences, University of Tokyo,

5-1-5 Kashiwanoha, Kashiwa, Chiba 277-8563, Japan

e-mail: okada@sml.k.u-tokyo.ac.jp

\begin{tabular}{|c|c|}
\hline $\mathbf{u}$ & Macroscopic displacement vector \\
\hline$\{\mathbf{u}\}$ & $\begin{array}{l}\text { Macroscopic structure nodal displacement } \\
\text { vector }\end{array}$ \\
\hline$\left\{\mathbf{u}^{e}\right\}$ & $\begin{array}{l}\text { Macroscopic structure nodal displacement } \\
\text { vector per element }\end{array}$ \\
\hline $\mathbf{w}$ & $\begin{array}{l}\text { Periodic component of the microscopic } \\
\text { displacement vector }\end{array}$ \\
\hline$\{\mathbf{w}\}$ & $\begin{array}{l}\text { Periodic component of the nodal } \\
\text { displacement vectors of all } \\
\text { microstructures }\end{array}$ \\
\hline$\left\{\mathbf{w}^{Q}\right\}$ & $\begin{array}{l}\text { Periodic component of the nodal } \\
\text { displacement vector of a single } \\
\text { microstructure }\end{array}$ \\
\hline$\left\{\mathbf{w}^{e}\right\}$ & $\begin{array}{l}\text { Periodic component of the nodal } \\
\text { displacement vector per element }\end{array}$ \\
\hline $\mathbf{F}$ & The deformation gradient tensor \\
\hline $\mathbf{Z}$ & The displacement gradient tensor \\
\hline $\mathbf{C}$ & The right Cauchy-Green tensor \\
\hline $\mathbf{E}$ & The Green-Lagrange strain tensor \\
\hline$\Pi$ & The first Piola-Kirchhoff stress tensor \\
\hline $\mathbf{I}$ & The identity tensor \\
\hline$I_{c}, I I_{c}, I I I_{c}$ & Principal invariants \\
\hline$J$ & Determinant $\mathbf{F}$ \\
\hline
\end{tabular}

\section{Introduction}

The door to petaflop computing has recently opened and meaningful applications for massively parallel computers are being sought. A multi-scale approach to biomechanical problems is consequential in the post-genome era and the homogenization method is going to play a more important role than ever before. The homogenization method is a mathematical modeling technique for efficiently analyzing inhomogeneous material with a periodic microstructure. In 
biomaterial, the periodicity hypothesized in the homogenization method is not strictly established. However, Terada et al. [1] have shown that an appropriate equivalent characteristic is obtained in material with an irregular microstructure by assuming a periodic boundary condition. Thus, it is possible to evaluate the effect of each component in the microstructure on the macroscopic behavior, if microstructure modeling is appropriate. The homogenization method for biomaterial was applied to bone by Hollister and Kikuchi [2], while a two-dimensional analysis of engineered tissue cells was conducted by Breuls et al. [3]. In an example using the heart, Krassowska et al. [4] applied the method to an excitation propagation phenomena. To investigate the effect of intracellular structure on heartbeat, the authors have developed the necessary finite element homogenization method, where the heart is the macrostructure and the cardiomyocyte the microstructure. Thus the problem inevitably becomes a large-scale one.

In the homogenization method two scales are introduced, namely, a scale for the unit period, and a scale for the whole material. By solving the governing equations for both scales with coupling, we can obtain the macroscopic characteristic as an equivalent homogeneous body and variable distribution from the microstructure. In the conventional nonlinear homogenization method [5,6], it is first necessary to calculate microscopic equilibrium and then the macroscopic tangential homogenization updates all quadrature points at every Newton-Raphson iteration, resulting in huge computational cost. Even with a high performance computer, the cost is prohibitive for practical large-scale problems. To reduce this computational cost, various techniques have been devised. These include, for example, the construction of a database with the homogenized properties [7], sensitivity analysis [8], Fast Fourier Transforms [9], and so on. In a previous work, we proposed a homogenization method using characteristic deformation mode superposition [10,11]. This is, however, an approximation method and the accuracy depends on the problem. We subsequently proposed a new algorithm that solves the microscopic equilibrium equation alternately with the solution of the mode superposition-based micro-macro coupled equation. In this algorithm, the equilibrium conditions for both the micro and macro structures are satisfied with far less computational cost. This method is applicable to microstructures composed of slightly incompressible and viscoelastic materials [12-15].

Looking at this method from the block LU factorization of the micro-macro coupled equation, we recognize that the Schur-Complement as the homogenized tangent stiffness, is ingeniously approximated with the aid of mode superposition. It is further beneficial to generalize this view, that is, to interpret the framework of the homogenization method with regard to the block LU factorization and investigate how the Schur-Complement can be approximated to reduce the

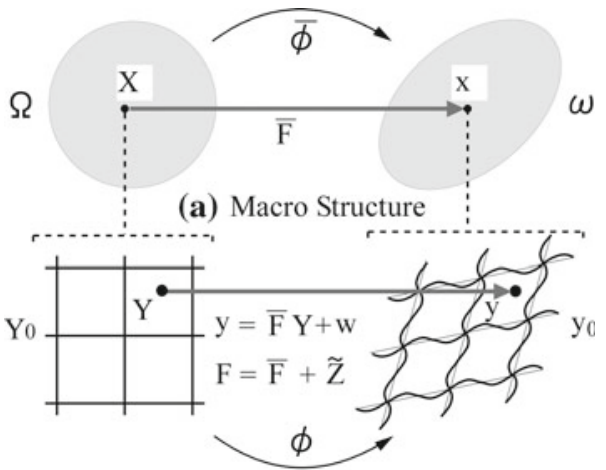

(b) Micro Structure

Fig. 1 Homogenization method for large deformation problems. a Macro structure. b Micro structure

computational cost whilst preserving the accuracy. Accordingly, a couple of approximation methods, i.e., a simple scheme to reuse the old characteristic deformation and the above-mentioned mode-superposition based method, are introduced in this paper. Then a simplified numerical example is solved using both the conventional homogenization algorithm and the algorithm originating from the block LU factorization, and the performance of each is discussed. Finally, a large-scale heart simulation using parallel computation is presented based on the proposed method.

\section{Homogenization method for finite deformation problem}

\subsection{Problem statement and geometric prospect}

We assume that the material in the body $(\Omega)$ reveals heterogeneity on a very fine scale and is characterized by the periodic distribution of a basic structural element $\left(Y_{0}\right)$ as shown in Fig. 1. To measure the changes in the spatial domains, we introduce two scales: a macro-scale $X \in \Omega$ and a micro-scale $Y \in Y_{0}$. Thus the actual domain can be regarded as the product space $\left(\Omega \times Y_{0}\right)$. In the subsequent development, the macroscopic quantity corresponding to the microscopic one is expressed with a bar symbol over the microscopic symbol. The following assumptions of homogenization are applied in the formulation of the homogenization method.

- A macrostructure that consists of a periodic microstructure can be considered to be an approximately equivalent homogeneous substance.

- A microstructure is infinitely fine compared with a macrostructure; the variable defined at each point of the macrostructure corresponds to the volume average of the variables in the microstructure. 
It is assumed that the deformation of the microstructure is linked to the local values of the macro continuum via

$\mathbf{y}=\overline{\mathbf{F}} \mathbf{Y}+\mathbf{w}$

where $\mathbf{y}$ and $\mathbf{Y}$ are position vectors defined on the microstructure [16].

The deformation consists of a homogeneous part $\overline{\mathbf{F}} \mathbf{Y}$ and a non-homogeneous superposed field w. Consequently, the following relationships exist between the microscopic and macroscopic deformation gradients.

$\mathbf{F}=\nabla_{Y} \mathbf{y}=\frac{\partial \mathbf{y}}{\partial \mathbf{Y}}=\overline{\mathbf{F}}+\tilde{\mathbf{Z}}$

$\overline{\mathbf{F}}=\nabla_{X} \mathbf{x}=\frac{\partial \mathbf{x}}{\partial \mathbf{X}}$

$\tilde{\mathbf{Z}}=\nabla_{Y} \mathbf{w}=\frac{\partial \mathbf{w}}{\partial \mathbf{Y}}$.

Thus increment and variation of the deformation gradients are represented, respectively, as

$$
\begin{aligned}
\Delta \mathbf{F} & =\Delta \overline{\mathbf{F}}+\Delta \tilde{\mathbf{Z}}=\Delta \overline{\mathbf{F}}+\nabla_{Y} \Delta \mathbf{w}, \\
\delta \mathbf{F} & =\delta \overline{\mathbf{F}}+\delta \tilde{\mathbf{Z}}=\delta \overline{\mathbf{F}}+\nabla_{Y} \delta \mathbf{w} .
\end{aligned}
$$

For the assumptions mentioned above, the macroscopic gradients are related via the volume averages

$\overline{\mathbf{F}}=\frac{1}{|V|} \int_{Y_{0}} \mathbf{F} d Y=\frac{1}{|V|} \int_{Y_{0}}(\overline{\mathbf{F}}+\tilde{\mathbf{Z}}) d Y=\overline{\mathbf{F}}+\frac{1}{|V|} \int_{Y_{0}} \tilde{\mathbf{Z}} d Y$,

where $V$ is the volume of the microstructure $Y_{0}$. Then, the fluctuation field $\mathbf{w}$ must satisfy the constraint

$\int_{Y_{0}} \tilde{\mathbf{Z}} d Y=\int_{Y_{0}} \frac{\partial \mathbf{w}}{\partial \mathbf{Y}} d Y=\int_{\partial Y_{0}} \mathbf{N} \otimes \mathbf{w} d S=\mathbf{0}$,

where $\mathbf{N}$ is an outward normal vector on the boundary $\partial Y_{0}$. This constraint is satisfied when $\mathbf{w}$ is periodic.

\subsection{Formulation of homogenization method and finite element discretization}

We now consider the equilibrium of material with a periodic microstructure, modeled by hyperelastic material. Using the principle of stationary potential energy, the equilibrium condition becomes a functional stationary problem. Under the homogenization assumptions, the macroscopic potential energy is related via the volume averages of the microscopic ones and the entire potential energy is defined by

$\Phi=\int_{\Omega} \frac{1}{|V|} \int_{Y_{0}} W d Y d X-\int_{\partial \Omega} \mathbf{t} \cdot \mathbf{u} d S$,

where $W$ is the strain energy function of the microstructure defined by the deformation gradient $\mathbf{F}$, and assuming conservative tractions. The stationary condition becomes

$\delta \Phi=\int_{\Omega} \frac{1}{|V|} \int_{Y_{0}} \delta \mathbf{F}: \Pi d Y d X-F_{\text {ext }}(\delta \mathbf{u})=0$,

$\Pi=\frac{\partial W}{\partial \mathbf{F}}$

$F_{\text {ext }}(\delta \mathbf{u})=\int_{\partial \Omega} \mathbf{t} \cdot \delta \mathbf{u} d S$.

A similar equation has been reported by Terada and Kikuchi [5] using two-scale convergence theory [17]. We have also shown a formulation based on the mixed variational principle with a perturbed Lagrange-multiplier [14]. By inserting Eq. (6) into Eq. (10), macro and micro equilibrium equations can be derived based on the defined space of the variation.

$\bar{G}=\int_{\Omega} \frac{1}{|V|} \int_{Y_{0}} \delta \overline{\mathbf{F}}: \Pi d Y d X-F_{\text {ext }}(\delta \mathbf{u})=0$,

$G=\int_{Y_{0}} \delta \tilde{\mathbf{Z}}: \Pi d Y=0$,

which achieves equilibrium under the given boundary condition in the macrostructure and self-equilibrium under a periodic boundary condition, Eq. (8), of the microscopic displacement in the microstructure. Thus the homogenization method simultaneously satisfies the two equilibrium conditions as described above. To solve the nonlinear equation, the Newton-Raphson method is employed. Then the standard linearization process in nonlinear finite element method provides the following linearized equations

$$
\begin{aligned}
\int_{\Omega} & \frac{1}{|V|} \int_{Y_{0}} \delta \mathbf{F}: \mathbf{A}: \Delta \mathbf{F} d Y d X \\
= & F_{\text {ext }}(\delta \mathbf{u})-\int_{\Omega} \frac{1}{|V|} \int_{Y_{0}} \delta \mathbf{F}: \Pi d Y d X, \\
\mathbf{A} & =\frac{\partial \Pi}{\partial \mathbf{F}} .
\end{aligned}
$$

Substituting Eqs. (5) and (6) into the above equation yields

$$
\begin{aligned}
& \int_{\Omega} \frac{1}{|V|} \int_{Y_{0}} s(\delta \overline{\mathbf{F}}+\delta \tilde{\mathbf{Z}}): \mathbf{A}:(\Delta \overline{\mathbf{F}}+\Delta \tilde{\mathbf{Z}}) d Y d X \\
& =F_{\text {ext }}(\delta \mathbf{u})-\int_{\Omega} \frac{1}{|V|} \int_{Y_{0}}(\delta \overline{\mathbf{F}}+\delta \tilde{\mathbf{Z}}): \Pi d Y d X .
\end{aligned}
$$

By finite element discretization using

$$
\begin{aligned}
\Delta \overline{\mathbf{F}} & =\left[\overline{B^{e}}\right]\left\{\Delta \mathbf{u}^{e}\right\}, \\
\Delta \tilde{\mathbf{Z}} & =\left[B^{e}\right]\left\{\Delta \mathbf{w}^{e}\right\},
\end{aligned}
$$


where $\left[B^{e}\right]$ is a shape function matrix, the left-hand side of Eq. (17) becomes

$$
\begin{aligned}
\left\{\delta \mathbf{w}^{Q}\right\} & \frac{1}{|V|} \int_{Y_{0}}\left[B^{e}\right]^{T}[A]\left[B^{e}\right] d Y\left\{\Delta \mathbf{w}^{Q}\right\} \\
& +\left\{\delta \mathbf{w}^{Q}\right\} \frac{1}{|V|} \int_{Y_{0}}\left[B^{e}\right]^{T}[A] d Y\left[\overline{B^{e}}\right]\left\{\Delta \mathbf{u}^{e}\right\} \\
& +\left\{\delta \mathbf{u}^{e}\right\}\left[\overline{B^{e}}\right]^{T} \frac{1}{|V|} \int_{Y_{0}}[A]\left[B^{e}\right] d Y\left\{\Delta \mathbf{w}^{Q}\right\} \\
& +\left\{\delta \mathbf{u}^{e}\right\}\left[\overline{B^{e}}\right]^{T} \frac{1}{|V|} \int_{Y_{0}}[A] d Y\left[\overline{B^{e}}\right]\left\{\Delta \mathbf{u}^{e}\right\} \\
= & \left\{\delta \mathbf{w}^{Q}\right\} \mathbf{K}_{w w}^{Q}\left\{\Delta \mathbf{w}^{Q}\right\}+\left\{\delta \mathbf{w}^{Q}\right\} \mathbf{K}_{w u}^{Q}\left\{\Delta \mathbf{u}^{e}\right\} \\
& +\left\{\delta \mathbf{u}^{e}\right\} \mathbf{K}_{u w}^{Q}\left\{\Delta \mathbf{w}^{Q}\right\}+\left\{\delta \mathbf{u}^{e}\right\} \mathbf{K}_{u u}^{Q}\left\{\Delta \mathbf{u}^{e}\right\},
\end{aligned}
$$

while the second term of the right-hand side of Eq. (17) becomes

$$
-\left\{\delta \mathbf{u}^{e}\right\}\left[\bar{B}^{e}\right]^{T} \frac{1}{|V|} \int_{Y_{0}}[\Pi] d Y-\left\{\delta \mathbf{w}^{Q}\right\} \frac{1}{|V|} \int_{Y_{0}}\left[B^{e}\right]^{T}[\Pi] d Y,
$$

at each quadrature point of the macrostructure. Symbol $Q$ denotes the quantity that is evaluated at a macroscopic quadrature point, while symbol $e$ denotes the quantity evaluated in the macroscopic element. By assembling these appropriately on the macro continuum, and considering the facultative variations, the following semi-positive definite symmetric matrix is obtained

$$
\left[\begin{array}{ll}
\mathbf{K}_{w w} & \mathbf{K}_{w u} \\
\mathbf{K}_{u w} & \mathbf{K}_{u u}
\end{array}\right]\left\{\begin{array}{c}
\Delta \mathbf{w} \\
\Delta \mathbf{u}
\end{array}\right\}=\left\{\begin{array}{c}
\mathbf{r}_{w} \\
\mathbf{r}_{u}
\end{array}\right\}
$$

where

$$
\begin{aligned}
& \mathbf{K}_{w w}=\int_{\Omega}\left(\frac{1}{|V|} \int_{Y_{0}}\left[B^{e}\right]^{T}[A]\left[B^{e}\right] d Y\right) d X \\
& \mathbf{K}_{w u}=\int_{\Omega}\left(\frac{1}{|V|} \int_{Y_{0}}\left[B^{e}\right]^{T}[A] d Y\right)\left[\overline{B^{e}}\right] d X \\
& \mathbf{K}_{u w}=\int_{\Omega}\left[\overline{B^{e}}\right]^{T}\left(\frac{1}{|V|} \int_{Y_{0}}[A]\left[B^{e}\right] d Y\right) d X \\
& \mathbf{K}_{u u}=\int_{\Omega}\left[\overline{B^{e}}\right]^{T}\left(\frac{1}{|V|} \int_{Y_{0}}[A] d Y\right)\left[\overline{B^{e}}\right] d X
\end{aligned}
$$

$$
\begin{aligned}
& \left\{\mathbf{r}_{w}\right\}=-\int_{\Omega}\left(\frac{1}{|V|} \int_{Y_{0}}\left[B^{e}\right]^{T}[\Pi] d Y\right) d X \\
& \left\{\mathbf{r}_{u}\right\}=F_{\text {ext }}-\int_{\Omega}\left[\overline{B^{e}}\right]^{T}\left(\frac{1}{|V|} \int_{Y_{0}}[\Pi] d Y\right) d X .
\end{aligned}
$$

The nonlinear homogenization method solves Eq. (22) for $\Delta \mathbf{u}$ and $\Delta \mathbf{w}$ under the given boundary condition for the macrostructure and the periodic boundary condition (Eq. 8) for microscopic displacement. The number of degrees of freedom (NDOF) of this matrix is (NDOF of macrostructure + quadrature point of macrostructure $\times$ NDOF of microstructure). An enormous computational cost is, however, required to solve a small-scale problem. Moreover, it is difficult to solve the form given in Eq. (8) due to memory limitations, and generally, a transformation into the weak form takes place as described below.

\subsection{Characteristic deformation}

In a nonlinear problem, to evaluate the response of a microstructure to macroscopic deformation in a similar way to that in a linear problem [18], we obtain the following equation by taking the derivative of Eq. (14) at each quadrature point and substituting Eqs. (5) and (16).

$$
\int_{Y_{0}} \delta \tilde{\mathbf{Z}}: \mathbf{A}: d \tilde{\mathbf{Z}} d Y=-\int_{Y_{0}} \delta \tilde{\mathbf{Z}}: \mathbf{A}: d \overline{\mathbf{F}} d Y
$$

Since the macroscopic deformation gradient is independent of the microscopic integration,

$\int_{Y_{0}} \delta \tilde{\mathbf{Z}}: \mathbf{A}: \frac{\partial \tilde{\mathbf{Z}}}{\partial \overline{\mathbf{F}}} d Y=-\int_{Y_{0}} \delta \tilde{\mathbf{Z}}: \mathbf{A}: \mathbf{I} d Y$,

where $\mathbf{I}$ is a fourth order identity tensor, and the microstructural response of the macroscopic deformation gradient becomes

$$
\begin{aligned}
& \frac{\partial \tilde{\mathbf{Z}}}{\partial \overline{\mathbf{F}}}=\nabla_{Y}\left(\frac{\partial \mathbf{w}}{\partial \overline{\mathbf{F}}}\right) \equiv-\nabla_{Y} \chi, \\
& \chi \equiv-\frac{\partial \mathbf{w}}{\partial \overline{\mathbf{F}}},
\end{aligned}
$$

where a third order tensor $\chi$ is the derivative of the microscopic displacement with respect to the macroscopic deformation gradient. This is referred to as the characteristic deformation for nonlinear problems. The equation above can be substituted into Eq. (30) yielding

$$
\int_{Y_{0}} \delta \tilde{\mathbf{Z}}: \mathbf{A}: \nabla_{Y} \chi d Y=\int_{Y_{0}} \delta \tilde{\mathbf{Z}}: \mathbf{A}: \mathbf{I} d Y .
$$


By finite element discretization, the gradient of $\chi$ becomes

$\nabla_{Y} \chi=\left[B^{e}\right]\left[\chi^{e}\right]$,

in a microscopic finite element. $\left[\chi^{e}\right]$ is the derivative of the microscopic displacement for each component of $\overline{\mathbf{F}}$; in other words, it is the matrix given below with nine kinds of characteristic deformation.

$\left[\chi^{e}\right]=\left[\begin{array}{cccc}\chi_{111}^{e} & \chi_{112}^{e} & \cdots & \chi_{133}^{e} \\ \chi_{211}^{e} & \chi_{212}^{e} & \cdots & \chi_{233}^{e} \\ \vdots & \vdots & \ddots & \vdots \\ \chi_{n 11}^{e} & \chi_{n 12}^{e} & \cdots & \chi_{n 33}^{e}\end{array}\right]$

where $n$ is the NDOF of one finite element of the microstructure. The matrix equation becomes

$\mathbf{K}_{\chi}\left[\chi^{Q}\right]=\left[\mathbf{r}_{\chi}\right]$,

$\mathbf{K}_{\chi}=\int_{Y_{0}}\left[B^{e}\right]^{T}[A]\left[B^{e}\right] d Y$,

$\left[\mathbf{r}_{\chi}\right]=\int_{Y_{0}}\left[B^{e}\right]^{T}[A][I] d Y$,

from Eq. (33) about one microstructure. $\left[\chi^{Q}\right]$ denotes that the quantity is evaluated at the macroscopic quadrature point. The value of $\left[\chi^{Q}\right]$, which is a (NDOF of the microstructure) row $\times 9$ column matrix, can be obtained by assembling $\left[\chi^{e}\right]$. $[I]$ is a ninth order identity matrix, consisting of nine column vectors $\left\{I_{i}\right\}(i=1 \ldots 9)$. Thus nine solutions can be obtained for the right-hand side of Eq. (38), one for each $\left\{I_{i}\right\}$ corresponding to a component of the deformation gradient. $\left[\chi^{Q}\right]$ can then be obtained by solving each different version of the right-hand side of the above equation.

Multiplying by $\left[\overline{B^{e}}\right]$ and dividing by $|V|$ on both sides, Eq. (36) yields

$\frac{1}{|V|} \mathbf{K}_{\chi}\left[\chi^{Q}\right]\left[\overline{B^{e}}\right]=\frac{1}{|V|}\left[\mathbf{r}_{\chi}\right]\left[\overline{B^{e}}\right]$.

Now, by using Eq. (20)

$$
\begin{aligned}
& \frac{1}{|V|} \mathbf{K}_{\chi}=\mathbf{K}_{w w}^{Q}, \\
& \frac{1}{|V|}\left[\mathbf{r}_{\chi}\right]\left[\overline{B^{e}}\right]=\mathbf{K}_{w u}^{Q},
\end{aligned}
$$

and thus,

$$
\mathbf{K}_{w w}^{Q}\left[\chi^{Q}\right]\left[\overline{B^{e}}\right]=\mathbf{K}_{w u}^{Q} .
$$

The total is obtained at all macroscopic quadrature points in respect of the above equation

$$
\begin{aligned}
& \mathbf{K}_{w w}[\chi][\bar{B}]=\mathbf{K}_{w u}, \\
& {[\bar{B}] \equiv \sum_{i}\left[\overline{B^{e}}\right],} \\
& {[\chi] \equiv \sum_{i}\left[\chi^{Q}\right],}
\end{aligned}
$$

where $[\chi]$ is a matrix consisting of (quadrature points of macrostructure $\times$ NDOF of microstructure) rows $\times 9$ columns.

The characteristic deformations are the deformation increments for unit macroscopic deformation gradients at a particular instant and these describe the material properties and strain distribution of the microstructure. Equation (29) can also be considered a linear approximation of the microscopic deformation. Therefore, the update of the microscopic deformation by

$\Delta \mathbf{w}=-\chi: \Delta \overline{\mathbf{F}}$

corresponds to the Forward Euler method for microscopic deformation from Eq. (32).

\subsection{Homogenization method using characteristic deformation mode superposition}

In the mode superposition homogenization method, the microscopic displacement increment is approximately obtained by the linear combination of the previously calculated characteristic deformation $0 \chi$ and the scaling factor from Eq. (46) as in [10]

$\Delta w_{k} \doteqdot-{ }_{0} \chi_{k p} Q \Delta \alpha_{p Q}$,

where $\alpha$ is the scaling factor for each mode. Inserting Eq. (47) into Eq. (22) yields the matrix

$$
\begin{aligned}
& {\left[\begin{array}{ll}
{[0 \chi]^{T}} & \mathbf{0} \\
\mathbf{0} & \mathbf{I}
\end{array}\right]\left[\begin{array}{ll}
\mathbf{K}_{w w} & \mathbf{K}_{w u} \\
\mathbf{K}_{u w} & \mathbf{K}_{u u}
\end{array}\right]\left[\begin{array}{ll}
{[0 \chi]} & \mathbf{0} \\
\mathbf{0} & \mathbf{I}
\end{array}\right]\left\{\begin{array}{l}
\Delta \alpha \\
\Delta \mathbf{u}
\end{array}\right\}} \\
& =\left[\begin{array}{ll}
{[0 \chi]^{T}} & \mathbf{0} \\
\mathbf{0} & \mathbf{I}
\end{array}\right]\left\{\begin{array}{l}
\mathbf{r}_{w} \\
\mathbf{r}_{u}
\end{array}\right\} \\
& {\left[\begin{array}{ll}
{[0 \chi]^{T} \mathbf{K}_{w w}[0 \chi]} & {[0 \chi]^{T} \mathbf{K}_{w u}} \\
\mathbf{K}_{u w}[0 \chi] & \mathbf{K}_{u u}
\end{array}\right]\left\{\begin{array}{l}
\Delta \alpha \\
\Delta \mathbf{u}
\end{array}\right\}=\left\{\begin{array}{l}
{[0 \chi]^{T} \mathbf{r}_{w}} \\
\mathbf{r}_{u}
\end{array}\right\},}
\end{aligned}
$$

where $[0 \chi]$ is the same kind of matrix as $[\chi]$. The above equation can be represented as

$$
\left[\begin{array}{ll}
\mathbf{K}_{\alpha \alpha} & \mathbf{K}_{\alpha u} \\
\mathbf{K}_{u \alpha} & \mathbf{K}_{u u}
\end{array}\right]\left\{\begin{array}{c}
\Delta \alpha \\
\Delta \mathbf{u}
\end{array}\right\}=\left\{\begin{array}{l}
\mathbf{r}_{\alpha} \\
\mathbf{r}_{u}
\end{array}\right\}
$$


where

$$
\begin{aligned}
& \mathbf{K}_{\alpha \alpha}=\int_{\Omega}\left(\frac{1}{|V|} \int_{Y_{0}}\left[0 \chi^{e}\right]^{T}\left[B^{e}\right]^{T}[A]\left[B^{e}\right]\left[0 \chi^{e}\right] d Y\right) d X, \\
& \mathbf{K}_{\alpha u}=-\int_{\Omega}\left(\frac{1}{|V|} \int_{Y_{0}}\left[0 \chi^{e}\right]^{T}\left[B^{e}\right]^{T}[A] d Y\right)\left[\overline{B^{e}}\right] d X, \\
& \mathbf{K}_{u \alpha}=-\int_{\Omega}\left[\overline{B^{e}}\right]^{T}\left(\frac{1}{|V|} \int_{Y_{0}}[A]\left[B^{e}\right]\left[0 \chi^{e}\right] d Y\right) d X, \\
& \mathbf{K}_{u u}=\int_{\Omega}\left[\overline{B^{e}}\right]^{T}\left(\frac{1}{|V|} \int_{Y_{0}}[A] d Y\right)\left[\overline{B^{e}}\right] d X, \\
& \left\{\mathbf{r}_{u}\right\}=F_{\text {ext }}-\int_{\Omega}\left[\overline{B^{e}}\right]^{T}\left(\frac{1}{|V|} \int_{Y_{0}}[\Pi] d Y\right) d X, \\
& \left\{\mathbf{r}_{\alpha}\right\}=\int_{\Omega}\left(\frac{1}{|V|} \int_{Y_{0}}\left[0 \chi^{e}\right]^{T}\left[B^{e}\right]^{T}[\Pi] d Y\right) d X .
\end{aligned}
$$

We obtain the matrix with unknowns $\Delta \alpha$ and $\Delta \mathbf{u}$. Because the NDOF of the matrix is reduced to (NDOF of macrostructure + quadrature point of macrostructure $\times 9$ ), significant computational cost is saved.

This technique is, however, an approximate means of achieving equilibrium in a range of displacements representing linear combinations of $\chi_{0}$, as it is clear from Eq. (56) that $\chi_{0}$ has an effect on the equilibrium. In this way, to approximate the deformation in limited deformation patterns, an approximation error is created depending on the analysis case [15].

\section{Algorithm for nonlinear homogenization method}

\subsection{Generalized algorithm}

In Eq. (22), $\{\Delta w\}$ can be statically condensed at the element quadrature point level and becomes

$\{\Delta \mathbf{w}\}=\mathbf{K}_{w w}^{-1}\left(\left\{\mathbf{r}_{w}\right\}-\mathbf{K}_{w u}\{\Delta \mathbf{u}\}\right)$.

$\{\Delta w\}$ vanishes when the above equation is substituted in the macroscopic equilibrium equation

$$
\left(\mathbf{K}_{u u}-\mathbf{K}_{u w} \mathbf{K}_{w w}^{-1} \mathbf{K}_{w u}\right)\{\Delta \mathbf{u}\}=\left\{\mathbf{r}_{u}\right\}-\mathbf{K}_{u w} \mathbf{K}_{w w}^{-1}\left\{\mathbf{r}_{w}\right\} .
$$

Now, the microscopic equilibrium hypothesized for $\overline{\mathbf{F}}$ at this time is

$\left\{\mathbf{r}_{w}\right\}=0$.
By using Eqs. (43) and (57), we obtain [5]

$$
\{\Delta \mathbf{w}\}=-\mathbf{K}_{w w}^{-1} \mathbf{K}_{w u}\{\Delta \mathbf{u}\}=-[\chi][\overline{\mathbf{B}}]\{\Delta \mathbf{u}\} .
$$

By using Eqs. (43), (57), and (58) we can represent

$\left(\mathbf{K}_{u u}-\mathbf{K}_{u w}[\chi][\overline{\mathbf{B}}]\right)\{\Delta \mathbf{u}\}=\left\{\mathbf{r}_{u}\right\}$,

where $\left(\mathbf{K}_{u u}-\mathbf{K}_{u w}[\chi][\overline{\mathbf{B}}]\right)$ is called the homogenized tangent stiffness.

In differential equation form, from Eqs. (14), (17), and (31), this becomes

$$
\begin{gathered}
\int_{\Omega} \delta \overline{\mathbf{F}}:\left(\frac{1}{|V|} \int_{Y_{0}}\left(\mathbf{A}:\left(\mathbf{I}-\nabla_{Y} \chi\right)\right) d Y\right): \Delta \overline{\mathbf{F}} d X \\
=\overline{\mathbf{F}}_{\text {ext }}-\int_{\Omega} \delta \overline{\mathbf{F}}:\left(\frac{1}{|V|} \int_{Y_{0}} \Pi d Y\right) d X .
\end{gathered}
$$

Microscopic equilibrium, Eq. (59),

$r_{w}=\int_{Y_{0}} \delta \tilde{\mathbf{Z}}: \Pi d Y=\mathbf{0}$,

is a prerequisite of the above equation. To satisfy this nonlinear prerequisite, a Newton-Raphson iteration is needed. Thus the above equation is linearized with respect to $\mathbf{w}$ while $\overline{\mathbf{F}}$ is fixed, and then discretized by the finite element method using Eq. (19). At each quadrature point, the linearized selfequilibrated equation becomes

$\int_{Y_{0}}\left[B^{e}\right]^{T}[A]\left[B^{e}\right] d Y\left\{\Delta \mathbf{w}^{Q}\right\}=-\int_{Y_{0}}\left[B^{e}\right]^{T}[\Pi] d Y$.

In the generalized algorithm, it is necessary to compute three different calculations in each iteration.

1. Update macroscopic tangential homogenization $\chi$ using Eq. (36).

2. Solve microscopic equilibrium problem, Eq. (63), and obtain the convergence solution $\{\mathbf{w}\}$ while $\overline{\mathbf{F}}$ is fixed.

3. Solve the linearized macroscopic equilibrium equation, Eq. (61), to obtain $\{\mathbf{u}\}$.

Processes 1 and 2 need to be solved at all quadrature points of the macrostructure and it is known that this contributes the most to the calculation load [6]. These processes require prohibitive computational cost and actual numerical simulation is difficult.

\subsection{Block LU factorization algorithm}

We now present an algorithm that decreases the residual of each scale simultaneously using the block LU factorization 
algorithm without microscopic convergence in the macroscopic iteration as in the conventional algorithm. By block LU factorization, Eq. (22) becomes

$$
\left[\begin{array}{ll}
\mathbf{K}_{w w} & \mathbf{0} \\
\mathbf{K}_{u w} & \mathbf{S}
\end{array}\right]\left[\begin{array}{ll}
\mathbf{I} & \mathbf{K}_{w w}^{-1} \mathbf{K}_{w u} \\
\mathbf{0} & \mathbf{I}
\end{array}\right]\left\{\begin{array}{c}
\Delta \mathbf{w} \\
\Delta \mathbf{u}
\end{array}\right\}=\left\{\begin{array}{l}
\mathbf{r}_{w} \\
\mathbf{r}_{u}
\end{array}\right\}
$$

where $\mathbf{S}$ is called the Schur-Complement. Referring to Eq. (43),

$$
\begin{aligned}
\mathbf{S} & =\mathbf{K}_{u u}-\mathbf{K}_{u w} \mathbf{K}_{w w}^{-1} \mathbf{K}_{w u} \\
& =\mathbf{K}_{u u}-\mathbf{K}_{u w}[\chi][\overline{\mathbf{B}}] \\
& =\mathbf{K}_{u u}-[\overline{\mathbf{B}}]^{T}[\chi]^{T} \mathbf{K}_{w w}[\chi][\overline{\mathbf{B}}],
\end{aligned}
$$

which corresponds to the homogenized tangent stiffness of Eq. (61). The solution process for Eq. (65) is composed of the forward substitution

$$
\left[\begin{array}{ll}
\mathbf{K}_{w w} & \mathbf{0} \\
\mathbf{K}_{u w} & \mathbf{S}
\end{array}\right]\left\{\begin{array}{c}
\Delta \tilde{\mathbf{w}} \\
\Delta \tilde{\mathbf{u}}
\end{array}\right\}=\left\{\begin{array}{c}
\mathbf{r}_{w} \\
\mathbf{r}_{u}
\end{array}\right\}
$$

and the backward substitution

$$
\left\{\begin{array}{c}
\Delta \tilde{\mathbf{w}} \\
\Delta \tilde{\mathbf{u}}
\end{array}\right\}=\left[\begin{array}{ll}
\mathbf{I} & \mathbf{K}_{w w}^{-1} \mathbf{K}_{w u} \\
\mathbf{0} & \mathbf{I}
\end{array}\right]\left\{\begin{array}{c}
\Delta \mathbf{w} \\
\Delta \mathbf{u}
\end{array}\right\}
$$

Then, from Eqs. (43), (67), and (68),

$$
\begin{aligned}
& \mathbf{K}_{w w}\{\Delta \tilde{\mathbf{w}}\}=\left\{\mathbf{r}_{w}\right\}, \\
& \mathbf{S}\{\Delta \mathbf{u}\}=\left\{\mathbf{r}_{u}\right\}-\mathbf{K}_{u w}\{\Delta \tilde{\mathbf{w}}\}, \\
& \{\Delta \mathbf{w}\}=\{\Delta \tilde{\mathbf{w}}\}-\mathbf{K}_{w w}^{-1} \mathbf{K}_{w u}\{\Delta \mathbf{u}\} \\
& =\{\Delta \tilde{\mathbf{w}}\}-[\chi] \Delta \overline{\mathbf{F}},
\end{aligned}
$$

hold. The calculation process is described below.

1. Solve linearized microscopic equilibrium equation, Eq. (69), to obtain $\{\Delta \mathbf{w}\}$.

2. Solve linearized macroscopic equilibrium equation, Eq. (70), to obtain $\{\Delta \mathbf{u}\}$. Note that this equation is equivalent to Eq. (58).

3. Update $\{\Delta \mathbf{w}\}$ using Eq. (71). We have already obtained $[\chi]$ from the calculation of $\mathbf{S}$.

Three matrices, namely the micro, macro, and SchurComplement, need to be solved at each iteration. Although the computational cost of this algorithm is expected to be lower than that of the generalized algorithm since equilibrium of the microstructure is not required at every iteration, the Schur-Complement update is still relatively expensive as described above. A similar algorithm for a differential equation using the Block-Newton method has been proposed by Yamada and Matsui [19].
3.3 Approximation of schur-complement in micro-macro coupled equation

In the block LU factorization algorithm described in the previous subsection, the linearized equations for the microstructure are solved first and then the increment for the macro-displacements is solved using the Schur-Complement that reflects the micro-macro interactions. These two steps essentially define the computational cost because the third step can be solved efficiently by reusing the characteristic deformation that has already been computed in the evaluation of the Schur-Complement in the second step. Although the macroscopic equilibrium conditions must be satisfied as well as the microscopic ones by making a convergence judgment for the residuals, the Schur-Complement is a tangent stiffness for prediction and does not influence the equilibrium directly. Hence, one of the requirements for efficient nonlinear homogenization algorithms is to approximate the Schur-Complement effectively. In this regard, we propose the two algorithms given below.

A simple method is to approximate the Schur-Complement using the previously calculated characteristic deformation $0 \chi$ as in

$$
\begin{aligned}
\mathbf{S} & =\mathbf{K}_{u u}-\mathbf{K}_{u w}[\chi][\overline{\mathbf{B}}] \\
& \doteqdot \mathbf{K}_{u u}-\mathbf{K}_{u w}[0 \chi][\overline{\mathbf{B}}] \\
& =\mathbf{K}_{u u}-[\overline{\mathbf{B}}]^{T}[\chi]^{T} \mathbf{K}_{w w}[0 \chi][\overline{\mathbf{B}}],
\end{aligned}
$$

that is,

$[\chi]^{T} \mathbf{K}_{w w}[\chi] \doteqdot[\chi]^{T} \mathbf{K}_{w w}[0 \chi]$,

is employed from Eq. (66). Henceforth, this method is referred to as the modified block LU factorization algorithm (MBFA). The other approach is to make use of the aforementioned Mode Superposition method in the approximation of the Schur-Complement. The calculation process and an interpretation thereof are given below.

1. Solve the linearized microscopic equilibrium equation, Eq. (69), to obtain $\{\Delta \tilde{\mathbf{w}}\}$.

2. From Eq. (50), solve the mode superposition-based linearized micro-macro coupled equation

$$
\left[\begin{array}{ll}
\mathbf{K}_{\alpha \alpha} & \mathbf{K}_{\alpha u} \\
\mathbf{K}_{u \alpha} & \mathbf{K}_{u u}
\end{array}\right]\left\{\begin{array}{c}
\Delta \alpha \\
\Delta \mathbf{u}
\end{array}\right\}=\left\{\begin{array}{c}
\mathbf{r}_{\alpha} \\
\mathbf{r}_{u}
\end{array}\right\}
$$

to obtain $\{\Delta \mathbf{u}\}$. Then, $\mathbf{S}$ is approximated by the range of the mode superposition method and the update of $\mathbf{S}$ can be omitted.

3. The assumption of the mode superposition method follows from Eqs. (46), (47), and (71), and $\{\Delta \mathbf{w}\}$ is updated using 


$$
\{\Delta \mathbf{w}\}=\{\Delta \tilde{\mathbf{w}}\}-[0 \chi]\{\Delta \alpha\} .
$$

The meaning of the algorithm is now given. Block LU factorization is applied to Eq. (74) yielding

$$
\begin{aligned}
& \mathbf{K}_{\alpha \alpha}\{\Delta \tilde{\alpha}\}=\left\{\mathbf{r}_{\alpha}\right\}, \\
& \left(\mathbf{K}_{u u}-\mathbf{K}_{u \alpha} \mathbf{K}_{\alpha \alpha}^{-1} \mathbf{K}_{\alpha u}\right)\{\Delta \mathbf{u}\}=\left\{\mathbf{r}_{u}\right\}-\mathbf{K}_{u \alpha}\{\Delta \tilde{\alpha}\}, \\
& \{\Delta \alpha\}=\{\Delta \tilde{\alpha}\}-\mathbf{K}_{\alpha \alpha}^{-1} \mathbf{K}_{\alpha u}\{\Delta \mathbf{u}\} .
\end{aligned}
$$

In Eq. (77), the macroscopic displacement is updated using

$$
\left(\mathbf{K}_{u u}-\mathbf{K}_{u \alpha} \mathbf{K}_{\alpha \alpha}^{-1} \mathbf{K}_{\alpha u}\right) \text {, }
$$

which can be considered an approximation of the homogenized tangent stiffness. Then, using Eqs. (43) and (66), the Schur-Complement becomes

$$
\begin{aligned}
\mathbf{S}= & \mathbf{K}_{u u}-\mathbf{K}_{u w}[\chi][\overline{\mathbf{B}}] \\
= & \mathbf{K}_{u u}-\mathbf{K}_{u w}[\chi]\left([\chi]^{T} \mathbf{K}_{w w}[\chi]\right)^{-1}\left([\chi]^{T} \mathbf{K}_{w w}[\chi]\right)[\overline{\mathbf{B}}] \\
= & \mathbf{K}_{u u}-\mathbf{K}_{u w}[\chi]\left([\chi]^{T} \mathbf{K}_{w w}[\chi]\right)^{-1}[\chi]^{T} \mathbf{K}_{w u} \\
\doteqdot & \mathbf{K}_{u u}-\mathbf{K}_{u w}[0 \chi]\left([0 \chi]^{T} \mathbf{K}_{w w}[0 \chi]\right)^{-1}[0 \chi]^{T} \mathbf{K}_{w u} \\
= & \mathbf{K}_{u u}-[\overline{\mathbf{B}}]^{T}[\chi]^{T} \mathbf{K}_{w w}[0 \chi] \\
& \times\left([0 \chi]^{T} \mathbf{K}_{w w}[0 \chi]\right)^{-1}[0 \chi]^{T} \mathbf{K}_{w w}[\chi][\overline{\mathbf{B}}] \\
= & \mathbf{K}_{u u}-\mathbf{K}_{u \alpha} \mathbf{K}_{\alpha \alpha}^{-1} \mathbf{K}_{\alpha u},
\end{aligned}
$$

that is,

$$
\begin{aligned}
& {[\chi]^{T} \mathbf{K}_{w w}[\chi] \doteqdot[\chi]^{T} \mathbf{K}_{w w}[0 \chi]} \\
& \quad \times\left([0 \chi]^{T} \mathbf{K}_{w w}[0 \chi)^{-1}[0 \chi]^{T} \mathbf{K}_{w w}[\chi],\right.
\end{aligned}
$$

is employed. In the mode superposition method, since the homogenized tangent stiffness corresponds to the exact value with the mode updated at every Newton-Raphson iteration as described above, this method can give an approximate mean of the homogenized tangent stiffness by decreasing the number of times that the mode is updated. This method is referred to as the mode superposition algorithm (MSA).

\section{Numerical examples}

\subsection{Comparison of computational costs and convergence properties}

With respect to computational cost and convergence, which are of interest to us, we now compare the four methods introduced in the previous section, namely, the generalized algorithm (GA), block LU factorization algorithm (BFA), modified block LU factorization algorithm (MBFA) and the algorithm using mode superposition (MSA). Detailed algorithms for the parallel computation of each of the methods are given in Fig. 2. The GA calculates the microscopic equilibrium at each quadrature point in every iteration. In contrast to the GA, the BFA, MBFA, and MSA decrease the residual

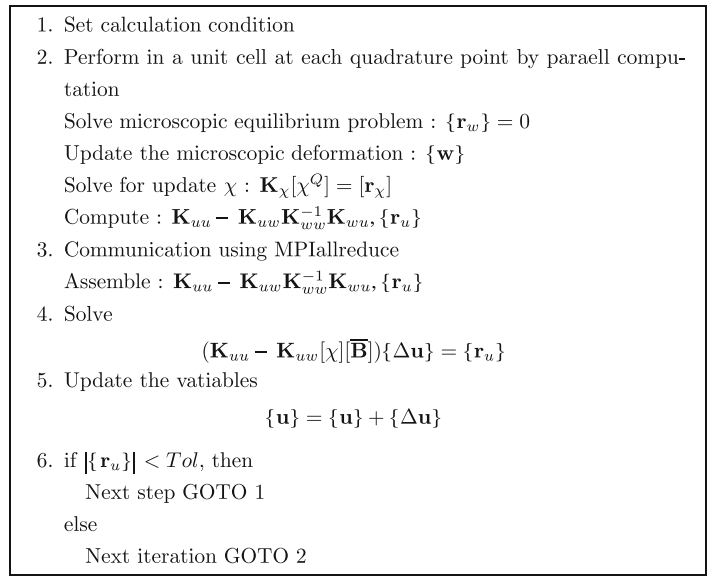

(a)

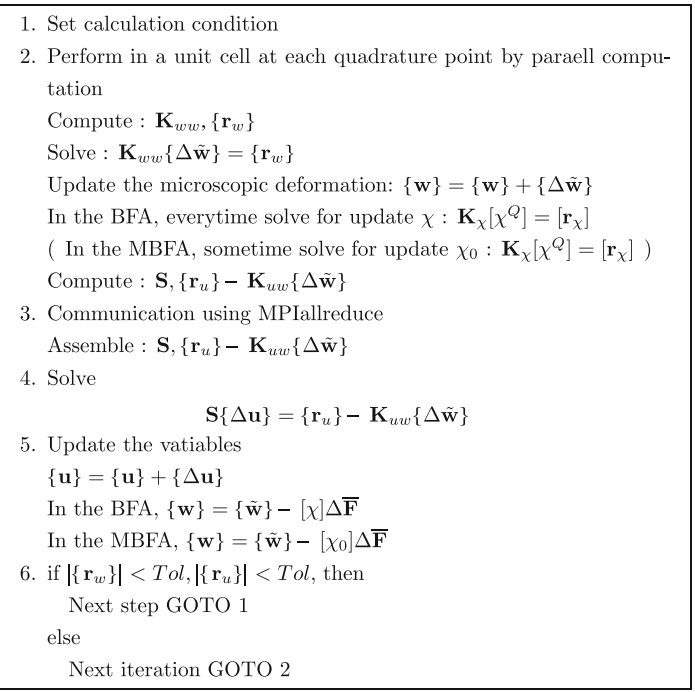

(b)

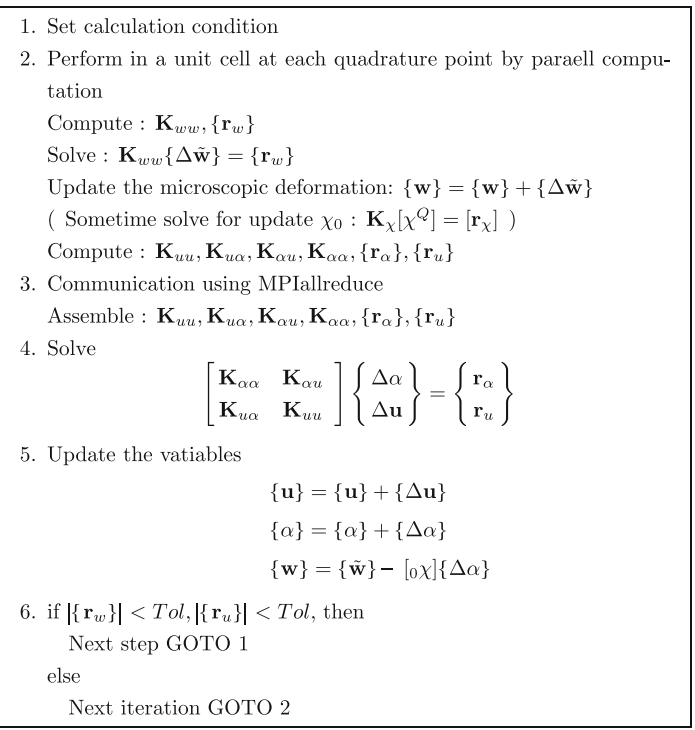

(c)

Fig. 2 Algorithms for parallel computation using the message passing interface (MPI). a Algorithms for GA. b Algorithms for BFA and MBFA. c Algorithms for MSA 


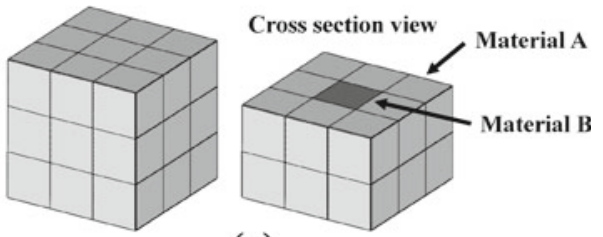

(a)

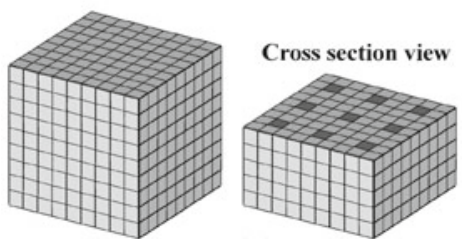

(b)

Fig. 3 FE models used in the calculation time evaluation. a 64 nodes, 27 elements (minimum size). b 1000 nodes, 729 elements

Table 1 Material constants for the models used in the calculation time evaluation

\begin{tabular}{lcc}
\hline & $E[\mathrm{kPa}]$ & $v$ \\
\hline Material A in Fig. 3 & 0.1 & 0.3 \\
Material B in Fig. 3 & 10.0 & 0.3 \\
\hline
\end{tabular}

of both scales simultaneously. Moreover, in the MBFA and MSA the straightforward update of $\mathrm{S}$ is avoided. We performed a $20 \%$ tensile test using the mesh of the block shown in Fig. 3 as the microstructure, and a single 8-node element as the macrostructure. The minimum size of the mesh is constructed from $27(3 \times 3 \times 3)$ elements as shown in Fig. 3a, with the stiffness of the center element different from the rest.

The NDOF is adjusted by adding the same number of minimum units in each direction (e.g. Fig. 3b). The St. Venant hyperelastic material is used for the constitutive equations

$$
\begin{aligned}
& W=\frac{1}{2} \lambda(t r \mathbf{E})^{2}+\mu \mathbf{E}: \mathbf{E}, \\
& \mathbf{S}=\frac{\partial W}{\partial \mathbf{E}}=(\lambda \mathbf{I} \otimes \mathbf{I}+2 \mu \mathbf{l}): \mathbf{E}=\mathbf{C}: \mathbf{E}, \\
& l_{i j k l}=\delta_{i k} \delta_{j l}, \\
& \lambda=\frac{E v}{(1+v)(1-2 v)}, \quad \mu=G=\frac{E}{2(1+v)},
\end{aligned}
$$

where $\mathbf{I}$ is a second order identity tensor, $\lambda$ and $\mu$ are Lame constants, and $E$ and $v$ are, respectively, Young's modulus and Poisson's ratio. $\mathbf{C}$ is a fourth order constant elasticity tensor and the relationship between $\boldsymbol{S}$ and $\boldsymbol{E}$ is linear. The material constants are given in Table 1. If the material constants are assumed to be uniform in the microstructure, the solutions obtained by these methods agree completely with the theoretical solution in the case of infinitesimal deformation. We have thus confirmed the correctness of the formulations

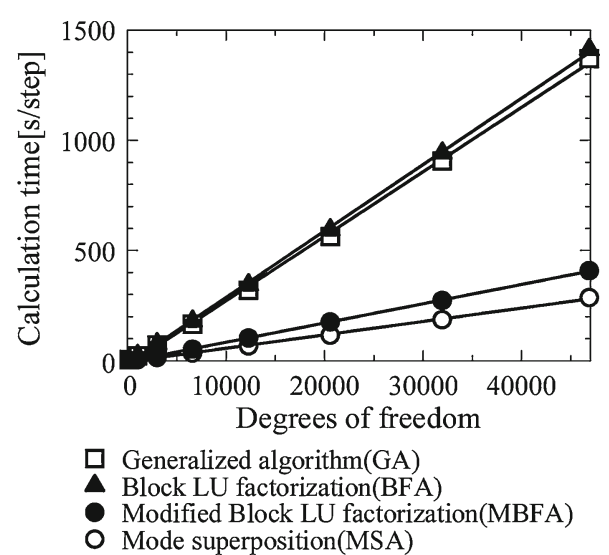

(a)

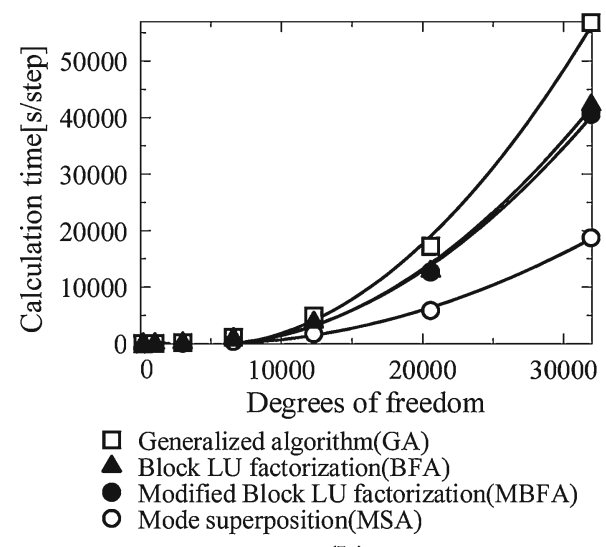

(b)

Fig. 4 Comparison of calculation time. a Iterative solver. b Direct solver

and computer programs. The relationship between calculation time and NDOF of the microstructure for each algorithm is depicted in Fig. 4 for cases where an iterative solver (ILU preconditioned GMRES method) or direct solver (skyline method) are used. The breakdown of calculation time and number of iterations in each process, where the NDOF of the microstructure is 31,944 , are given in Table 2 . Convergence is judged to occur when the root sum square reaches $1 \times 10^{-10}$ times the initial value. As shown in Fig. 4, the calculation time of the BFA using an iterative solver slightly exceeds that of the GA. In the BFA, equilibrium of the microstructure is not required within an iteration for the sake of computational efficiency, but this may result in more iterations compared with the GA. Table 2 illustrates this, in that the BFA requires 5 iterations whereas the GA requires 4 . We need to calculate the characteristic deformation to update the Schur-Complement; this means that the nine different right-hand sides of Eq. (33) must be solved in the case of the iterative solver. Consequently, the increased number of iterations results in a deterioration in the performance of the BFA. If a direct solver is used instead, the result of the LU decomposition 
Table 2 Calculation time and number of iterations in each process

\begin{tabular}{llll}
$\begin{array}{l}\text { Calculation } \\
\text { of } \chi(\mathrm{s})\end{array}$ & $\begin{array}{l}\text { Calculation of } \\
\text { equilibrium in the } \\
\text { microscopic model (s) }\end{array}$ & Total (s) & $\begin{array}{l}\text { No. of } \\
\text { iterations }\end{array}$ \\
\hline
\end{tabular}

a. Iterative solver

$\begin{array}{lrrrr}\text { GA } & 670 & 211 & 906 & 4 \\ \text { BFA } & 805 & 107 & 942 & 5 \\ \text { MBFA } & 17 & 195 & 273 & 11 \\ \text { MSA } & 21 & 113 & 186 & 5\end{array}$

b. Direct solver

\begin{tabular}{lrrrr} 
GA & 19,816 & 36,949 & 56,791 & 4 \\
BFA & 23,689 & 18,492 & 42,212 & 5 \\
MBFA & 451 & 40,050 & 40,557 & 11 \\
MSA & 457 & 18,237 & 18,746 & 5 \\
\hline
\end{tabular}

of the stiffness matrix can be reused in the computation of the characteristic deformation, and as a result, the computational cost of the BFA is $20 \%$ less than that of the GA, despite the BFA using one more iteration than the GA. On the other hand, the MBFA is much faster than both the BFA and the GA when an iterative solver is used, although in the case of a direct solver there is almost no difference between the MBFA and BFA. This is because the calculation cost of the mode update in the BFA is similar to that of the increased iterations in the MBFA. However, convergence in the MBFA is slow as shown in Fig. 5 and it is anticipated that for strong nonlinear problems, convergence may not be achieved at all. Here, the characteristic deformation was approximated by that obtained at the beginning of the analysis. Convergence can be improved by incorporating more frequent updates, at the expense of CPU time. Compared with these methods, the MSA always exhibits excellent speed and convergence. The advantage of the MSA is twofold. First, the approximation of the Schur-Complement is more accurate than in

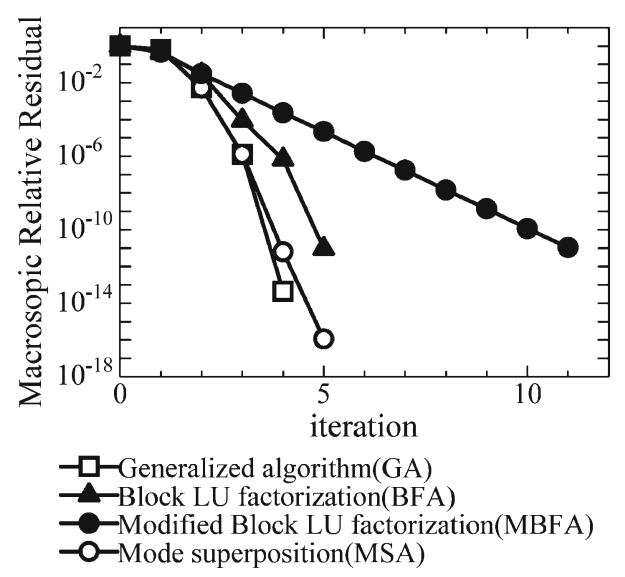

Fig. 5 Comparison of convergence property the MBFA, i.e., Eq. (80) gives a superior approximation than Eq. (72). Second, in an iteration of the MSA, the microscopic equation is solved in Eq. (75) in terms of $\Delta \alpha$ as well as in Eq. (74) with Eq. (69). These advantages should contribute to an accelerated convergence of the microscopic equation as well as the macroscopic equation. Although the St. Venant hyperelastic material assumed here has relatively weak nonlinearity, the number of iterations does not increase up to a large deformation range, even with the initial characteristic deformation being used for $0 \chi$. However, a periodic update of $0 \chi$ is required in the case of strong nonlinear material, such as an elasto-plastic material. If $0 \chi$ is updated at every iteration, the homogenized tangent stiffness corresponds to the exact value as described in Eq. (80).

\subsection{Application to ventricle-cardiomyocyte analysis with parallel computation}

As seen in Table 2, most of the calculation cost relates to the microscopic equilibrium (Eq. (69)) when the MBFA or MSA is used. In addition, as the NDOF of the microscopic model becomes larger, the computational cost for the macroscopic model becomes more negligible. It is therefore crucial to decrease the time for microscopic calculation in real problems. Since Eq. (69) holds independently at each macroscopic quadrature point, parallel computation is effective in the homogenization method. In other words, microscopic models can be distributed equally to the available cores, and this directly accelerates the microscopic calculation according to the number of cores. Moreover, since the memory is shared by fewer microscopic models in the parallel computation, a greater NDOF of the microstructure can be handled.

Figures 6 and 7 show a simplified human cardiomyocyteventricle model, to which we have applied the MSA. As shown on the left and in the center of Fig. 7 a simplified cardiomyocyte model is constructed with extracellular and intracellular matrices and gap junctions. The total NDOF is 20385. If the models are arrayed periodically in the three directions as depicted on the right of the figure, a fairly accurate imitation of a microgram of real tissue is obtained. The

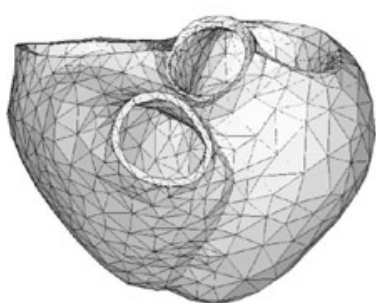

(a)

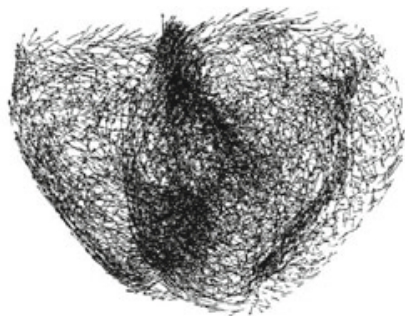

(b)
6554 elements, 1 quadrature point

Fig. 6 FE meshes of ventricles as macroscopic model. a FE mesh. b Fiber orientation 


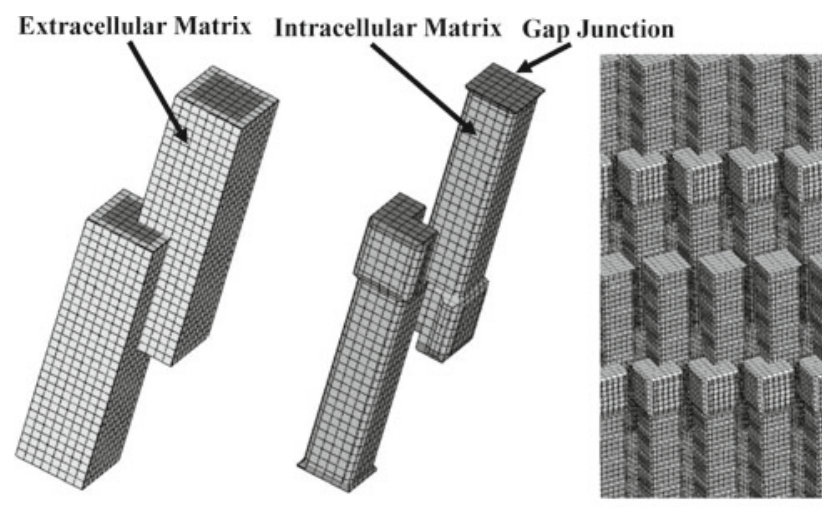

5736 elements, 6795 nodes

Fig. 7 FE meshes of cardiomyocytes as microscopic model

Table 3 Material constants for the cell model

\begin{tabular}{llll}
\hline & $C_{1}$ & $C_{2}$ & $\kappa$ \\
\hline Gap Junction & $1 \times 10^{5}$ & $1 \times 10^{5}$ & $2 \times 10^{5}$ \\
Intracellular Matrix & $1 \times 10^{4}$ & $1 \times 10^{4}$ & $2 \times 10^{4}$ \\
Extracellular Matrix & $1 \times 10^{3}$ & $1 \times 10^{3}$ & $2 \times 10^{3}$ \\
\hline
\end{tabular}

Mooney-Rivlin material using reduced invariants is adopted for the constitutive equation, with the material constants listed in Table 3.

$W=C_{1}\left(\tilde{I}_{c}-3\right)+C_{2}\left(\tilde{I}_{c}-3\right)+\frac{\kappa}{2} U^{2}$

$\tilde{I}_{c}=\frac{I_{c}}{I I I_{c}^{1 / 3}}, \quad \tilde{I}_{c}=\frac{I I_{c}}{I I I_{c}^{2 / 3}}$

$U=J-1$,

where $U$ is the volumetric strain energy function and $\kappa$ is the bulk modulus. The ventricle model is constructed based on CT imaging, with fiber directions distributed from -90 to 60 degrees, relative to the plane perpendicular to the long axis of the ventricle. The fiber direction denotes the long axis (z-axis) of the cardiomyocyte model, and therefore, proper rotation is taken into consideration for each micromodel. In this model, the intracellular matrix is defined as a function of a parameter that represents the excitation of the myocyte, and this parameter is varied at every time step to represent the transient contraction force. The homogenization method is applied to 6,554 elements covering the greater region of the ventricle, whereas the conventional Mooney-Rivlin hyperelastic constitutive law is assumed for the limited regions at the base and apex. To reduce the computational cost, a single myocyte (micromodel) is assigned to each finite element of the ventricle, i.e., 6,554 cardiomyocyte models are embedded in the ventricle model. The total NDOF, including those of the ventricles, amounts to 133,609,263.

The computer used was an IBM Blade Center consisting of 336 Power6 $(4.0 \mathrm{GHz})$ processors. Considering the size of
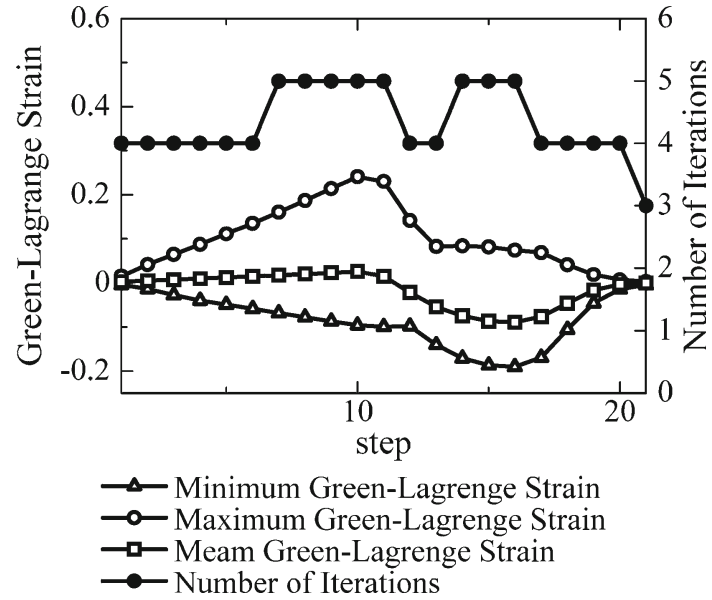

Fig. 8 Green-Lagrenge strains and nunber of iterations at each step

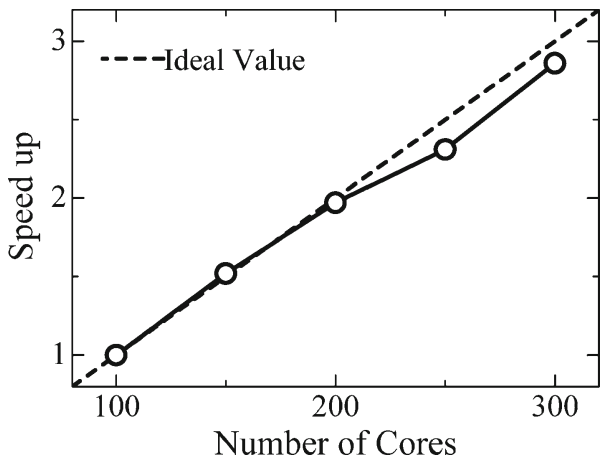

Fig. 9 Speed up in parallel computation

the problem, convergence was deemed to occur when the root sum square of the macro and micro residuals was $1 \times 10^{-5}$ times less than the initial value. Figure 8 shows the maximum, the minimum and the mean Green-Lagrange strains throughout the ventricle model, and the number of iterations of the MSA at each time step. The strains are measured in the fiber direction. Although the maximum and the minimum strains reach $\pm 20 \%$, an excellent convergence property was observed. Figure 9 shows the resulting scalability, by depicting the speed up rate relative to a hundred-core computation. As shown in this figure, satisfactory parallel performance was obtained by the proposed algorithm. The deformations of the ventricle and a representative myocyte in the diastole and the systole are exemplified in Fig. 10. The CPU time for the completion of a cardiac cycle was about $24 \mathrm{~h}$ when using 300 cores. The proposed method thus allows us to deal with large-scale problems.

\section{Conclusion}

To reduce the computational cost of the nonlinear homogenization method, the theoretical framework was reassessed 

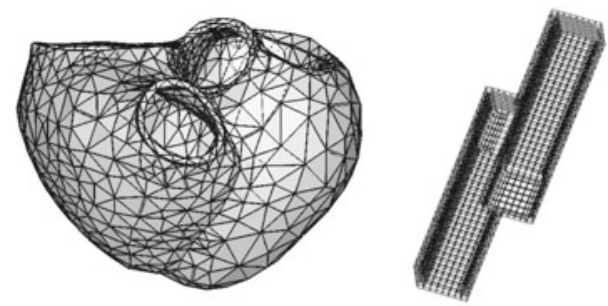

Diastole
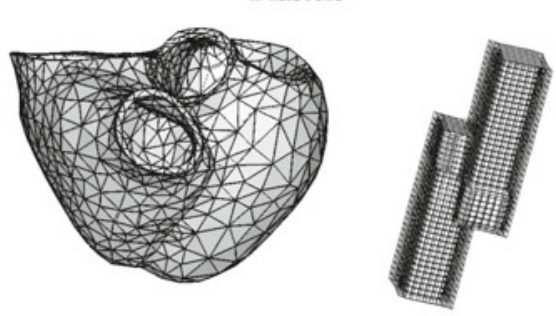

Systole

Fig. 10 Deformations of macro and microstructures in diastole and systole

from the perspective of block LU factorization of the micromacro coupled equation. Based on the relation between the characteristic deformation and the Schur-Complement as the homogenized tangent stiffness, a couple of approximation methods were introduced, namely, a simple scheme to reuse the old characteristic deformation (MBFA) and a sophisticated method based on the mode-superposition method (MSA) developed by our group. It is noted that accuracy is preserved in these approximation methods by incorporating the equilibrium conditions in both scales. Then, using a simplified FE model, the conventional algorithm (GA), a relative algorithm originating from the block LU factorization (BFA), the MBFA, and the MSA were compared and discussed. Of these methods, the MSA was found to be the best. Then, using the MSA, a large-scale human ventricle-cardiomyocyte simulation was performed on an IBM Blade Center consisting of 336 Power6 processors, and good parallel performance was demonstrated. We plan to use the proposed homogenization algorithm in a whole-heart simulation on a massively parallel computer in the near future.

Acknowledgments Our current research on homogenization method stems from Reference [10] coauthored by Hirohisa Noguchi. We again recognize his enthusiasm and contribution in a broad range of computational mechanics. This work was supported by Core Research for Evolutional Science and Technology, Japan Science and Technology Agency.

Open Access This article is distributed under the terms of the Creative Commons Attribution Noncommercial License which permits any noncommercial use, distribution, and reproduction in any medium, provided the original author(s) and source are credited.

\section{References}

1. Terada K, Hori M, Kyoya T, Kikuchi N (2000) Simulation of the multi-scale convergence in computational homogenization approaches. Int J Solids Struct 37:2285-2311

2. Hollister SJ, Kikuchi N (1992) Comparison of homogenization and standard mechanics analyses for periodic porous composites. Comput Mech 10:73-95

3. Breuls RGM, Sengers BG, Oomens CWJ, Bouten CVC, Baaijens FPT (2002) Predicting local cell deformations in engineered tissue constructs: a multilevel finite element approach. ASME J Biomech Eng 124:198-207

4. Krassowska W, Pilkington TC, Ideker RE (1990) Potential distribution in three-dimensional periodic myocardium: Part I. Solution with two-scale asymptotic analysis. IEEE Trans Biomed Eng 37:252-266

5. Terada K, Kikuchi N (2001) A class of general algorithms for multi-scale analyses of heterogeneous media. Comput Methods Appl Mech Eng 190:5427-5464

6. Matsui K, Terada K, Yuge K (2004) Two-scale finite element analysis of heterogeneous solids with periodic microstructures. Comput Struct 82:593-606

7. Takano N, Ohnishi Y, Zako M, Nishiyabu K (2001) Microstructure-based deep-drawing simulation of knitted fabric reinforced thermoplastics by homogenization theory. Int J Solids Struct 38:6333-6356

8. Rohan E (2003) Sensitivity strategies in modelling heterogeneous media undergoing finite deformation. Math Comput Simul 61:261270

9. Moulinec H, Suquet P (1998) A numerical method for computing the overall response of nonlinear composites with complex microstructure. Comput Methods Appl Mech Eng 157:69-94

10. Yamamoto M, Hisada T, Noguchi H (2001) Homogenization method for large deformation problem by using characteristic deformation mode superposition. Trans Jpn Soc Mech Eng 67:1877-1884 (in Japanese)

11. Yamamoto M, Hisada T (2002) A homogenization method for nearly incompressible materials by using characteristic deformation mode superposition. Trans Jpn Soc Mech Eng A 45:596-602

12. Okada J, Hisada T (2006) Homogenization method for mixed finite element analysis of incompressible hyper-elastic materials. In: JSCES e06: Proceedings of the 11th computational engineering conference, Osaka, Japan, pp 79-82 (in Japanese)

13. Okada J, Hisada T (2006) Homogenization method for mixed finite element analysis of incompressible viscoelastic materials. In: Proceedings of the 19th JSME computational mechanics conference, Nagoya, Japan, pp 45-46 (in Japanese)

14. Okada J, Hisada T (2009) Study on compressibility control of hyperelastic material for homogenization method using mixed finite element analysis. J Comput Sci Technol 3(1):89-100

15. Okada J, Washio T, Hisada T (2009) Nonlinear homogenization algorithms with low computational cost. J Comput Sci Technol 3(1):101-114

16. Miehe C, Schroder J, Schotte J (1999) Computational homogenization analysis in finite plasticity. Simulation of texture development in polycrystalline materials. Comput Methods Appl Mech Eng 171:387-418

17. Allaire G(1992) Homogenization and two-scale convergence. SIAM J Math Anal 23:1482-1518

18. Terada K, Kikuchi N (2003) Introduction to the method of homogenization. Maruzen (in Japanese)

19. Yamada T, Matsui K (2007) Numerical procedure for nonlinear multiscale problems based on block Newton method. Proc Conf Comput Eng Sci 12:309-312 (in Japanese) 